\title{
The Influence of Implicit and Explicit Biofeedback in First-Person Shooter Games
}

\author{
Kai Kuikkaniemi, Toni Laitinen, \\ Marko Turpeinen, Timo Saari, Ilkka Kosunen \\ Helsinki Institute for Information Technology \\ P.O. Box 9800, 02015 TKK, Finland \\ $<$ firstname $>$.<lastname $>$ @ hiit.fi
}

\begin{abstract}
To understand how implicit and explicit biofeedback work in games, we developed a first-person shooter (FPS) game to experiment with different biofeedback techniques. While this area has seen plenty of discussion, there is little rigorous experimentation addressing how biofeedback can enhance human-computer interaction. In our two-part study, $(\mathrm{N}=36)$ subjects first played eight different game stages with two implicit biofeedback conditions, with two simulation-based comparison and repetition rounds, then repeated the two biofeedback stages when given explicit information on the biofeedback. The biofeedback conditions were respiration and skin-conductance (EDA) adaptations. Adaptation targets were four balanced player avatar attributes. We collected data with psychophysiological measures (electromyography, respiration, and EDA), a game experience questionnaire, and game-play measures.
\end{abstract}

According to our experiment, implicit biofeedback does not produce significant effects in player experience in an FPS game. In the explicit biofeedback conditions, players were more immersed and positively affected, and they were able to manipulate the game play with the biosignal interface. We recommend exploring the possibilities of using explicit biofeedback interaction in commercial games.

\section{Author Keywords}

Games, playing, affective computing, biosignals, biofeedback, implicit biofeedback, explicit biofeedback.

\section{ACM Classification Keywords}

H1.2. Models and Principles: User/Machine Systems (Human factors). H5.2. Information interfaces and presentation: User Interfaces (Theory and methods).

\section{General terms}

Design, experimentation, human factors.

Permission to make digital or hard copies of all or part of this work for personal or classroom use is granted without fee provided that copies are not made or distributed for profit or commercial advantage and that copies bear this notice and the full citation on the first page. To copy otherwise, or republish, to post on servers or to redistribute to lists, requires prior specific permission and/or a fee.

CHI 2010, April 10-15, 2010, Atlanta, Georgia, USA

Copyright 2010 ACM 978-1-60558-929-9/10/04_..\$10.00.

\author{
Niklas Ravaja \\ Center for Knowledge and Innovation Research \\ P.O. Box 1210, 00101 Helsinki, Finland \\ niklas.ravaja@hse.fi
}

\begin{abstract}
INTRODUCTION
Emotions or emotion-related variables play a critical role in gaming behavior $[5,7,10,16]$. People seek, and are eager to pay for, games that elicit strong emotional experiences and enjoyment. Therefore, one of the major design goals for video game developers is to optimize emotional responses or response patterns in the gaming experience.
\end{abstract}

In recent years, there has also been a growing interest in new interaction modalities to expand the user experience in gaming. Traditional interaction techniques (e.g. mouse, keyboard, joystick, and gamepad) have been complemented and sometimes replaced by new interaction tools and devices: sensors and visual processing that track body movement (e.g., the Wii remote ${ }^{1}$ and Microsoft Project $\mathrm{Natal}^{2}$ ), specialized input devices (e.g., steering wheels, weapons, and musical instruments), and devices that can capture physiological signals (e.g., the Wii vitality sensor ${ }^{3}$ ).

Although various definitions of emotions have been proposed, the most general is that emotions are biologically based action dispositions that have an important role in the determination of behavior [e.g., 9]. Most theorists also endorse the view that emotions comprise three components: subjective experience (e.g., feeling joyous), expressive behavior (e.g., smiling), and physiological activation (e.g., sympathetic arousal) [13].

Psychophysiological signals can be effectively used in systems built for automatic recognition of human emotion [9, 13]. A biofeedback system captures data about the user's relevant psychological responses (or the realization of the parameters of set psychological states) with different methods of measurement, processes the information, and loops it back to the system as an input. Biosensing systems include electroencephalogram (EEG), electromyography (EMG), electrocardiogram (ECG), electrodermal activity measurement (EDA), and measurement of respiration change (RESP).

\footnotetext{
${ }^{1}$ See http://www.nintendo.com/wii/what/controllers\#remote

${ }^{2}$ See http://www.xbox.com/en-US/live/projectnatal/

${ }^{3}$ See http://www.engadget.com/2009/06/02/nintendo-wii-vi tality-sensor-detects-your-pulse/
} 
Use of biosignals can be divided between explicit and implicit feedback systems. The concept of explicit biofeedback originates from the field of medicine, with the purpose of making the subjects more aware of their bodily processes by displaying information about them in a clear and easily perceivable way. In the case of implicit biofeedback, the system modulates its behavior according to the biosignals of the subject, who may not even become consciously aware of the feedback but still senses it at a subconscious level. Thus the subject in the case of explicit biofeedback has direct and conscious control over the application while with implicit biofeedback the application is controlled indirectly according to the affective signals (or genuine emotions).

Our definition of implicit biofeedback is similar to the notion of affective feedback [1]. Gilleade et al. [6] refine this definition of affective feedback by stating that with affective feedback, in contrast to traditional biofeedback, the person may not even be aware that physiological state is being sensed, since the intent is to capture normal affective reactions.

Separating explicit and implicit biofeedback is not completely problem-free, since the line between them might become blurred if the user in the case of implicit biofeedback starts learning how the system works and thereby gains control over it. In such a case, implicit biofeedback becomes explicit.

Explicit biofeedback in the context of biofeedback gaming is used to control the game straightforwardly and explicitly. Informing the player that his level of arousal controls the speed of his character in a game (when it actually does) is an example of explicit biofeedback. A case where the player does not know that his level of arousal, for example, affects the game's difficulty is an example of implicit biofeedback.

Although research has been conducted into the utilization of biofeedback in the context of gaming [e.g., 1, 3, 11, 15], these studies tend to explore only the affordances of design instead of thoroughly analyzing the fundamental design issues related to the general structure of biofeedback. In practice, other studies have utilized biofeedback in event-based or step-function-based adaptation. We chose to focus on biofeedback with real-time dynamic connectivity in order to maximize the effects of the biosignal responses produced by the game.

The first-person shooter (FPS) is a well-established and popular video game genre, which centers the game play on weapon-based combat through the first-person perspective. In FPS games, the player experiences the action through the eyes of a protagonist. Thus, they can provide an intuitive context for experimenting with different mappings between a player's physiological state and the actions in the game.

The goal of this research was to find out what influence implicit and explicit biofeedback mechanisms have on the FPS game playing experience. We consider this topic as a useful component in gaining better understanding of the broader design issues in implementing biofeedback interaction systems. It is a popular trend that various game mechanics are utilized in utility solutions like education, simulation, exercising, group work and design. For this reason we believe that our work in biofeedback interaction will find applications in a broad range of domains.

\section{THE EMOSHOOTER PLATFORM}

The Emoshooter platform, which we used for performing our experiment, was assembled around an open-source graphics engine called OGRE. We chose to develop the game ourselves, which allowed us to manipulate all aspects of game play. Also, ready-made game-development-specific features were not considered as important as the flexible graphics-rendering capabilities that OGRE had. In addition to the graphics engine, the platform includes a physics engine called Open Dynamics Engine (ODE), OpenAL as its $3 \mathrm{D}$ audio engine, and various content production tools.

The biofeedback part of the game platform is composed of traditional game-development-specific features with integrated support for different types of biofeedback interaction. The biofeedback-related components are the biosignal processor, implemented as a separate component from the game application layer, and the biofeedback controller, integrated into the game application layer. Figure 1 shows the composition of the platform's architecture.

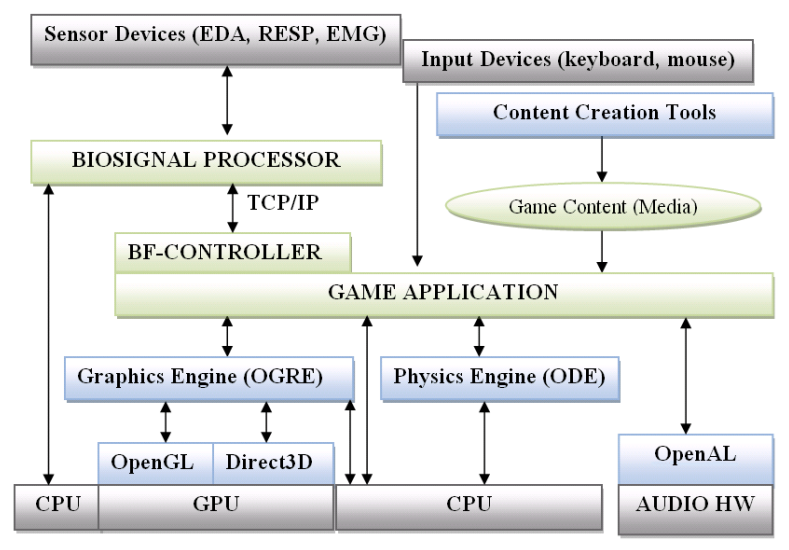

Figure 1: Emoshooter platform

A mobile physiological data acquisition system (Varioport-B) was used to measure the players' physiological responses. Although the system can record EEG (eight channels), facial EMG (three channels), ECG, EDA, RESP, and acceleration, only EDA, RESP, and EMG were used in our experiment. An Intel Pentium Core 2 Duo desktop computer with an NVIDIA GeForce 7950 GX2 graphics accelerator was used to run both the game application and the biosignal processor in our experiment.

\section{Biosignal Processor}

The main functions of the biosignal processor are to support the measuring hardware (Varioport-B), logging EDA, RESP, and EMG biosignal values as text files and to process the EDA and RESP biosignals into a form that the game application can use. The biosignal processor was implemented as a separate component from the game 
application. Communication between the two components was implemented via TCP/IP to enable removing the processing load from the CPU that ran the game application.

In the early prototyping phase, it was noticed that without proper calibration or normalization of biosignal data the biofeedback procedures would not work properly and the biofeedback effects implemented would break the game balance. It is very hard to implement stable and robust calibration, so we chose to normalize the biosignals. This was done according to a simple formula in which BSi is the current sample value of the biosignal (coming from the measuring hardware), BSmin is the biosignal's minimum value, and BSmax is the maximum value of the biosignal (see Figure 2).

Normalizing RESP and EDA biosignal sources is not completely trivial. There is a lot of difference in breathing rhythm and amplitude, which makes normalized signals ultimately insensitive in long-term studies. In practice, BSmin is reached when the lungs are empty and BSmax when they are full. Normalizing EDA is even harder because there are no obvious rules when the signal reaches maximum or minimum. For these reasons, we decided to use continuously updated local minima and maxima. For the EDA and RESP biofeedback sources, the normalization procedure is the same. In the biosignal processor, different biosignals are encapsulated in instances of a constant-length circular array class that keeps a record of the biosignal's local minimum and local maximum values. Every time a new sample value is inserted into the biosignal's array structure, a check is made of whether that value could be the signal's new local minimum or maximum. If the new sample value is less than the previously saved temporary minimum, a new temporary minimum is set. If the new sample value is greater than the previously saved temporary maximum, a new temporary maximum is set. Then, after $\mathrm{N}$ samples (we used a duration of six seconds), the saved temporary minimum and maximum values are set as the biosignal's new local minimum and maximum values by linearly interpolating from the old values to the new. Interpolation was used to avoid stepping effects in biofeedback procedures (and in game state changes). With this local normalization method, the effects of biofeedback procedures became more dynamic and responsive, and potentially more readily perceivable.

\section{Biofeedback Controller}

The biofeedback controller was implemented as a framework of related components in the game application, and the biofeedback procedure consisted of three distinct parts: biofeedback source, biofeedback transfer function, and biofeedback target. The biofeedback source corresponds to the type and value of the player state data encapsulated in different instances of the BiofeedbackValue class. Biofeedback target refers to the modulated game state variables also encapsulated in instances of the BiofeedbackValue class, this class being an abstract representation of both biofeedback source and biofeedback target objects and having member variables for the current value, minimum value, and maximum value for its state variable (player state or game state). The biofeedback transfer function realizes the game state modulations by using biofeedback source (and current game state) as its input and biofeedback target as its output. Biofeedback transfer functions are represented as instances of the BiofeedbackFunction class. Biofeedback procedures are represented as instances of the Biofeedback class, which has member variables for the biofeedback source object, biofeedback transfer function object, and biofeedback target object. Biofeedback interaction is then realized via various biofeedback procedures inserted into the biofeedback controller's list structure by the game system's logic and updated with every frame.

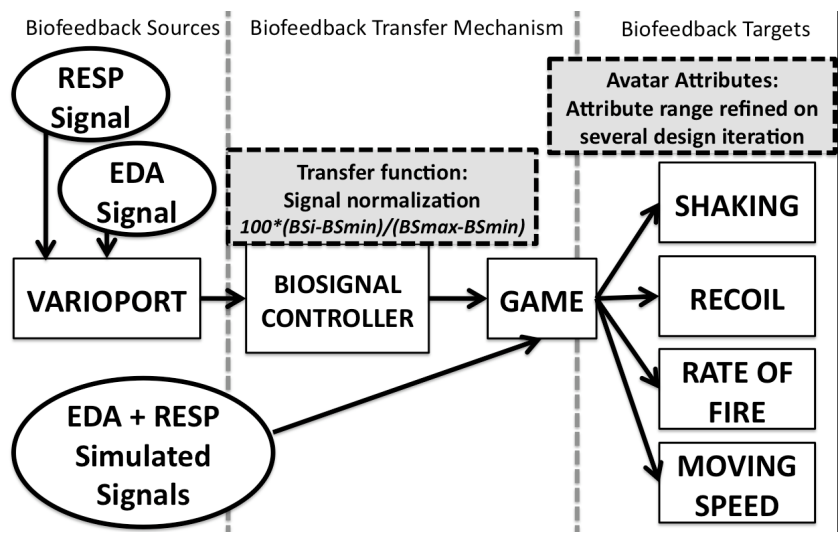

Figure 2: Adaptation mechanism

Figure 2 depicts the biofeedback adaptation mechanism from biofeedback sources via transfer mechanism to desired biofeedback targets, such as avatar attributes.

\section{EXPERIMENT DESIGN}

\section{Participants}

The 36 players recruited for the experiment were all over 18 years old, and most were experienced FPS players. All of the players were required to have at least some experience with FPS gaming. Almost all had an academic background.

At the beginning of the experiment, all players were asked questions regarding their gender, age, education, and gaming experience. There were three females and 33 males, all of age 18-35. Two players played games for 0 hours weekly, 21 played $1-5$ hours a week, 10 played 6-10 hours per week, and three played more than 10 hours. Eighteen players had 0 hours of FPS play weekly, 14 had 1-5 hours, and two each had 6-10 hours and more than 10 hours. The players self-evaluated their FPS experience on a scale of $1-7$, with six players scoring 2,10 scoring 3 , six scoring 4 , 11 scoring 5, and one each scoring 6 and 5.5. One (female) player did not evaluate herself.

\section{Design Rationale}

Several design choices were made during the experiment and prototype design. Ultimately, we chose an experiment setup with the following features: a within-subjects design; two distinct phases, with implicit biofeedback in phase 1 
(participants uninformed of biofeedback) and explicit biofeedback in phase 2 (participants informed of biofeedback); randomized conditions within first phase; restricted time in each condition; only one primary stimulus at the time; and balanced adaptation targets.

The experiment was conducted as a within-subjects design. The test conditions were implemented as different game stages, with the type of biofeedback interaction as a controlled variable. The dependent variable in the experiment was game experience in its different forms.

The weakness of a within-subject design is its "carryover effects." In general, carryover effects refer to unwanted effects within a test condition that are caused by previous test conditions. In the case of our biofeedback game experiment, the causes for carryover effects were practice (or experience obtained from playing the previous game stages), boredom, and fatigue. The players gain experience and get bored and fatigued when they play similar game stages over and over again. Because of the carryover effects, different game stages (test conditions) may be experienced differently only because their ordering is different. This was clearly undesirable in our experiment. Randomizing the order in which the conditions were played minimized the carryover effects. In order to increase the results' validity and further decrease carryover effects, there were two randomized rounds of repetition of the game stages in first phase. It is not practically possible to create implicit condition after explicit condition and for that reason there is no randomizing between phases.

Biofeedback processing can be conceptually divided into three distinct parts that can be varied: biofeedback source, biofeedback transfer function, and biofeedback target object. Our original plan was to test all three varieties of biofeedback processing. However, the play testing was very time-consuming, and only the biofeedback source could be varied while the transfer functions and biofeedback target objects were kept the same throughout the experiment.

EDA and RESP were selected as the biofeedback sources for the experiment. EMG was considered as a biofeedback source, but we decided not to use it, because it was hard to design a responsive EMG-based implicit adaptation. Intuitively, we also thought that EDA would be more difficult to manipulate than RESP.

To determine the impact of biofeedback interaction on the game playing experience, it was reasoned that the playing experience arising from biofeedback interaction (EDA-BF and RESP-BF) should be compared with that from similar play conditions with no biofeedback interaction (EDA-SIM and RESP-SIM). This was achieved via simulated transfer functions with sufficient similarity to their BF counterparts. With these conditions, it should be possible to separate the experience arising from pure aesthetics or changes in game controls from that produced by the biofeedback mechanisms.

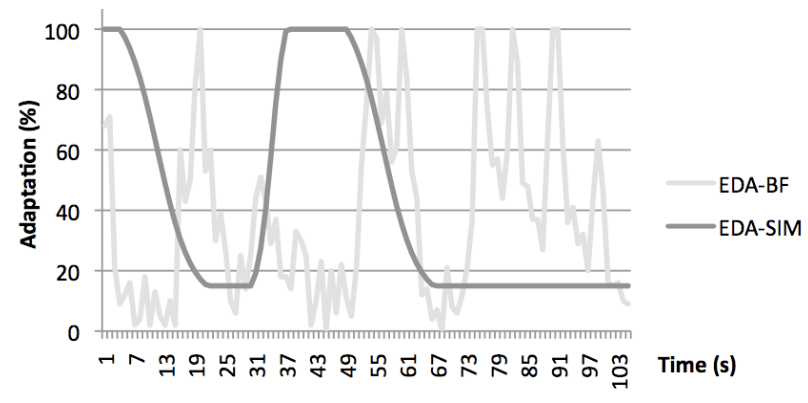

Figure 3: EDA-SIM and EDA-BF adaptation transfer function for an arbitrarily selected player

A piecewise-defined function was used to simulate the rise and fall of the EDA curve. Both functions were modified sigmoid functions. Figure 3 illustrates how EDA-SIM and EDA-BF adaptation transfer functions behave for a randomly selected player. It should be noted that some players had smoother EDA-BF than others. EDA-SIM is much smoother than EDA-BF. The EDA-SIM transfer function design was based on our experience from early prototyping.

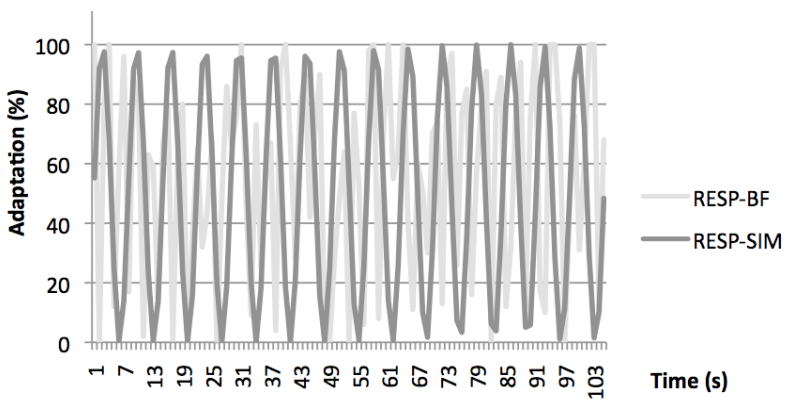

Figure 4: RESP-SIM and RESP-BF adaptation function

A sinusoidal function was used to simulate RESP, representing the continuous lung flow during game play. Figure 4 illustrates how the RESP-SIM and RESP-BF transfer function behaves. As shown, the RESP functions behave fairly similarly.

A few studies have explored the design possibilities for using biofeedback to enhance the game playing experience. In their work, Gilleade et al. [6] suggest three biofeedback game design patterns: assist me, challenge me, and emote $m e$. The first two patterns aim to modulate the game's difficulty level, while the third suggests that the aesthetics of the game could be regulated according to the current affective state of the player. Dekker et al. [3] implemented numerous biofeedback procedures in a biofeedback game targeting many attributes, including player character attributes, difficulty level, background music volume, shader effects, and game AI.

Rather than just proceeding to test modulations of some obvious features of background music or enhancing some visualizations of the game, for example, we propose a novel biofeedback game design pattern (or technique), character identification, that tries to address the common problem of player character identification in games. Player character 
identification is an important building block of game playing experience [8]. When players properly identify with their character, self-perception is changed. In an ideal situation, the players start to feel that they are one with their character and start to experience the emotions and the story in the way the designer had planned. Our assumption is that the character identification pattern is an effective way to modulate game play and has good potential to reveal the effects of biofeedback in comparison to using traditional (non-biofeedback) game-play mechanics, if there are any.

\section{Game Play}

Finding the right balance between game play and biofeedback for the experiment was problematic because robust experimental conditions required relatively simple game design. The instances of game mechanics that were modulated by biofeedback interaction were moving, aiming, and shooting (see Figure 2). The player character was able to move by walking, turning, and jumping. In the case of moving, the walking speed and turning speed were modulated. For aiming and shooting, the aiming direction, amount of recoil, and firing rate were modulated. Also the magnitude of shaking of the player character was continuously modulated with the biofeedback interaction. The continuous shaking deflected the position of the character slightly in a random direction. Unlike any other modulation, the magnitude of shaking modulation was perceptible all the time, even when the player was not employing any game mechanics. Besides making the firstperson view shake, the shaking of the character made walking and aiming more challenging. In addition, jumping strength and field of view modulations were implemented, but they were not used in play testing, because they interfered too much with the actual game play (moving and shooting). To avoid increasing the number of conditions (or game stages) in the play-testing procedure, all target attributes mentioned above were modulated and tested simultaneously during the same game stages.

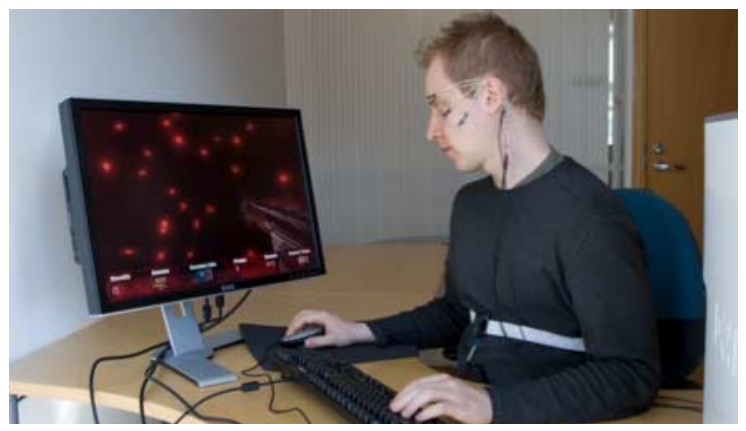

Figure 5: Game interface

Game controls were designed to be similar to those in typical FPS games where the player character is controlled with a keyboard and mouse. In addition to conventional interface devices, there were biosensors attached to the player before play began: two EDA electrodes were attached to the player's fingers, six EMG electrodes were attached to the face (two electrodes for each relevant face muscle), and a respiration band was placed around the player's chest (see Figure 5).

Game play was similar in each game stage. It was divided into two, alternating shooting modes: a sniper rifle mode (see Figure 6) and a machine gun mode (see Figure 7). In order to make the effects of biofeedback interaction perceptible in the game play, it was necessary for the players to use the game mechanics. Therefore, the game had to be designed in a way that persuaded players to use the game mechanics continuously.

In sniper mode, the game's goal was to aim at and shoot as many far-away enemies as possible. Far-away enemies stood still in a circle formation for 12 seconds, after which they merged to form one bigger and stronger enemy that started to move towards the player character. Once the bigger enemy reached the player character, it started circulating about it and shooting back, which initiated the machine gun phase.

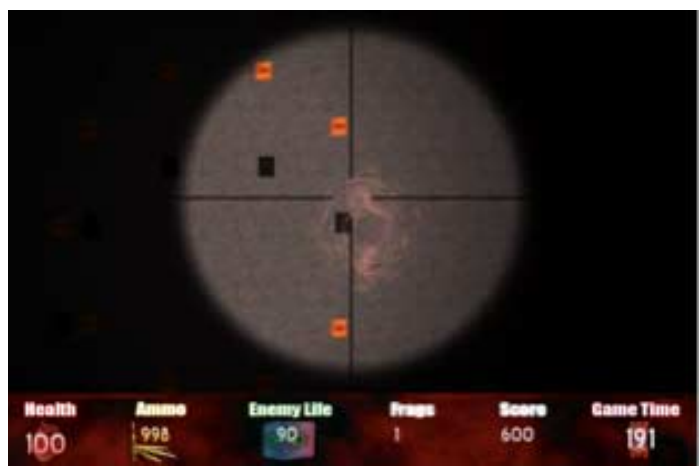

Figure 6: Sniper rifle mode

In machine gun mode, the game goals were to kill the circulating enemy by shooting it with a machine gun and to keep the player character unharmed by evading the enemy and its bullets and avoiding falling into the lava. The position information on the circulating enemy was enhanced for the player with a looping 3D sound effect and with a trailing tail particle effect. After 40 seconds, if the enemy was still alive, it made a kamikaze hit, killing both itself and the player character. When the enemy is killed, machine gun mode ends and sniper rifle mode restarts.

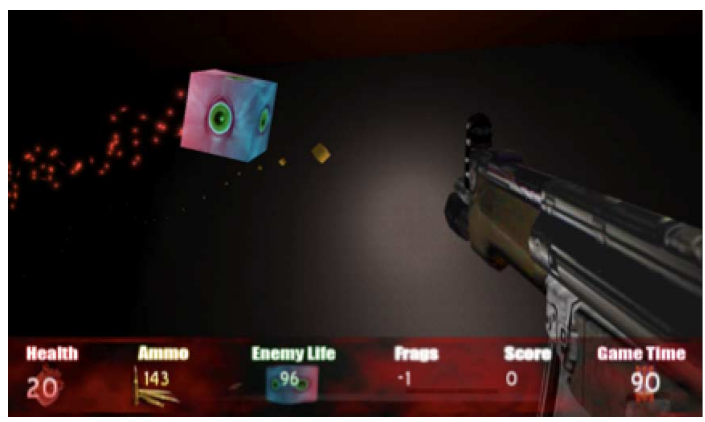

Figure 7: Machine gun mode 


\section{Game Environment}

Our experiment design assumes that the test conditions or game stages are the same for each subject. This means that the game stimuli (and game play) for the same game stages have to remain equal from repetition to repetition even when different people play the game stages. In the case of real-time FPS games, it is clearly impossible to produce completely identical play sessions for different players, but with the game play and game environment kept simple enough (and with time limits for game stages), this requirement could be at least roughly fulfilled. In order to allow extracting the players' reactions for the game, it was important also not to use too many parallel stimuli in the game. With too much use of parallel game stimuli (too many simultaneous visual and aural events, for example), it could become hard to tell what caused a particular response in the player and the effects of the biofeedback interaction could be lost in noise.

To obtain player state data resulting from the intended stimuli (game mechanics modulations) and not from unintended stimuli (player-defined goals, for example), the game environment was designed to be simple in such a way that it was not possible for the players to escape the planned condition. This choice supported the equality of stimuli among players and made the recorded results comparable. By our not including any hiding places or complicated tunnels in the level map, all the players' efforts could be directed to the continuous moving, aiming, and shooting that was necessary for the effects of the biofeedback interaction (or game mechanics modulations) to be perceivable.

The game environment has simulated physics with normal downwards gravitation and rigid body collisions. A looping ambient sound with echo was used to make the environment feel more spacious. The level map is a very large room that has three rectangular walking platforms in the middle of it. The sniper rifle was especially effective in this big room. At the beginning of each game stage, the player character starts at the edge of the middle platform. The smaller, spawning platforms are connected to the middle platform with two narrow bridges. With the bridges narrow enough, it is difficult for the player to stay on them while shaking a lot. That made the shaking a meaningful modulation from the game-play perspective. Enemies are spawned far from all platforms.

\section{Conditions}

There were two primary conditions in the game experiment: implicit biofeedback and explicit biofeedback. Within implicit game play, there were four sub-conditions, with biofeedback (using EDA-BF and RESP-BF) and respective simulation (EDA-SIM and RESP-SIM). In the explicit biofeedback condition, there was no simulation and were only respiration and EDA biofeedback sub-conditions.

The connection between the biofeedback source and the target attribute was made quite linear and easily noticeable.
When the current value of the biosignal (EDA or RESP) increased or decreased, the value of the target attribute followed. In modulation of the values of game mechanics attributes, it was important to keep the magnitude of the attributes in the proper range. Also, it was considered important that the players be able to notice the effects of the modulations during the game play session. Hence, the maximum magnitude for the game mechanics attributes (walking speed, turning speed, amount of shaking, rate of fire, and recoil) had to be set high enough. On the other hand, it was important for the game play that the maximum magnitude for the attributes not be too great, so that the game could be played without too much frustration.

During the implementation and pre-testing, it was noticed that with arbitrary game mechanics modulations the game's difficulty level was also likely to be affected. For example, if only the rate of fire was increased, the game became clearly easier. This was seen as problematic, since affecting the game's difficulty level would most likely also affect the playing experience and therefore interfere with the measurement of biofeedback experience. The purpose of the experiment was to measure the experience arising from the biofeedback interaction (or from the player state - game state connection), not from the modulation of the game's difficulty level. This problem was solved by programming the transfer functions in a way that preserved the game balance by keeping the total utility from all of the separate game mechanics modulations approximately invariable. For example, if one game mechanics modulation was set to increase the rate of fire when the player grew more aroused, another was set to increase the amount of recoil, preserving the game balance by making it harder to hit the target.

The overall effect of all the separate game mechanics modulations was also designed to be meaningful in the sense that it affected the game play and the player character properties in a manner that could be perceived as an exaggeration of the expressive behavior of the player character. When the player became aroused (when his EDA value rose) during game play, the character began to shake more, move more rapidly, and shoot more rapidly and with stronger recoil. When the player calmed down (when his EDA value fell), the player character became slower and steadier. In the case of the RESP biofeedback source, the modulations were the same. When the player breathed out, the character became faster but shakier. When the player inhaled, the player character became slower but steadier.

\section{Experiment Procedure}

To summarize our experiment procedure, Figure 8 illustrates how a single play-testing session was conducted.

The top-level goal of the game-play session was to get as many points as possible by playing 10 similar game stages, each with a duration of 200 seconds. After each game stage, a stage-specific high score list was displayed, from which the player could see his performance. In order to increase the motivation for the game play, all players were told that 
the player who gets the best overall high score after 10 played game stages wins five movie tickets.

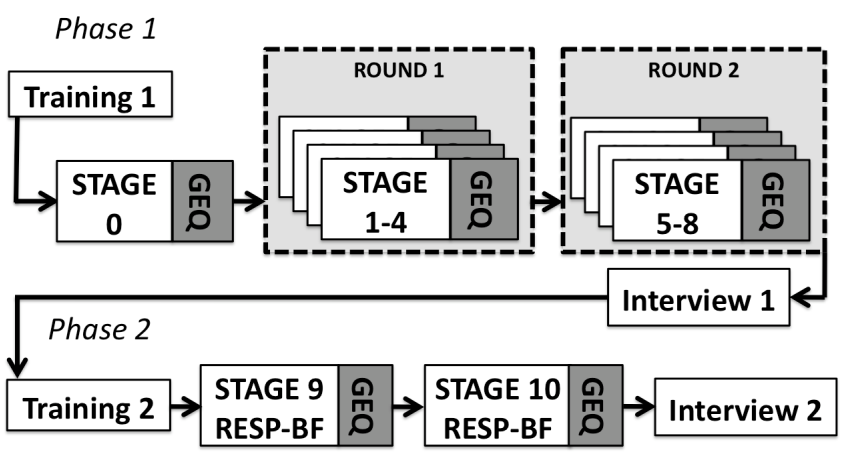

Figure 8: Experiment procedure

At the beginning of phase 1 , there was a training stage and a zero stage. In these two stages, the experimenters explained how the game works and how it should be played.

After each game stage, the player completed a game experience questionnaire $(\mathrm{GEQ})^{4}$ measuring various dimensions of playing experience. After the zero stage's GEQ was answered, the experimenters left the test room and the player was left alone to play the next eight game stages. In addition to the GEQ filled in after each condition (or game stage), game-play measures were gathered continuously.

The measurements recorded in real time were divided among three log files according to their type. Player state measures (EMG, EDA, and RESP) were recorded in biosignal log files by the biosignal processor with a sampling rate of 1,000 samples per second. Behavioral measures (such as mouse movement, amount of firing, and score), game state measures (e.g., stage beginning or a new ammo box being spawned), and game event measures (dying and killing) were logged with a sampling rate of 50 samples per second. We refer to these measures as game-play measures.

To make the results between stages comparable within the implicit phase (phase 1), we used two sets of randomized condition stacks: round 1 (stages 1-4) and round 2 (stages 5-8). During these stages, the players were not aware that the game was a biofeedback game that modulated certain features of game mechanics according to the physiological responses measured (EDA and RESP).

After the eight game stages of phase 1, the experimenters returned to the testing room and interviewed the player. At the end of the first interview, the player was told that the game being played is a biofeedback game that reacts to the

${ }^{4}$ The GEQ, developed by K. Poels, W. A. IJsselsteijn, and Y. A. W de Kort, of the Game Experience Lab in Eindhoven, The Netherlands (http://www.gamexplab.nl), is available on request. It is disseminated as a deliverable in the EU project Fun of Games (FUGA) and used in a variety of game studies globally. player's physiological responses by modulating certain features of game mechanics, depending on the measured responses. In phase 2, the player played stages nine and ten (explicit biofeedback). After the last game stage, the player was again interviewed and the experiment was ended.

\section{RESULTS}

Implicit Biofeedback (Phase 1)

In phase 1, players played in each condition (EDA-SIM, RESP-SIM, EDA-BF, and RESP-BF) twice without knowing that in the BF stages there was biofeedback interaction with the game. We implemented a manipulation check measure in interview 1 to confirm this. The manipulation check indicated that three players of the 36 , or $9 \%$, had some idea about the biofeedback and others were completely unaware of biofeedback manipulation. Hence, we can say that phase 1 represented an implicit biofeedback condition. According to our second interview, all subjects noticed biofeedback in phase 2. The RESP condition was obvious to all, but there was some uncertainty of the mechanics related to the EDA condition. We did not ask all players explicitly how they perceived the EDA condition in phase 2 .

Overall, the data were analyzed according to the Linear Mixed Models procedure in SPSS with restricted maximum likelihood estimation and a first-order autoregressive covariance structure for the residuals. Player ID was specified as the subject variable, and the stage number was specified as the repeated variable.

Stage number was selected as a factor, and a fixed-effects model that included stage number as the main effect was specified. Table 1 shows the relevant effects, including implicit biofeedback, and comparison between implicit and explicit biofeedback. We have used balls (0) to imply effect significance. One ball () means $\mathrm{p}<0.05$, two $(\boldsymbol{\bullet})$ means $\mathrm{p}<0.01$, and three (⿻)

Learning curve (difference between round 1 and round 2) and difference between EDA-BF and RESP-BF were the most significant effects within phase 1. Significant differences were found from questionnaire data and behavioral data but not in psychophysiology data. In learning curve effects, the competence increased (first round 2.3-3.0, second round 2.9-3.6), challenge decreased (first round 3.3-3.4, second round 3.0-3.1), tension decreased (first round 2.5-3.1, second round 2.2-2.4), negative affect increased, players scored better (used more sniper action and killed more enemies), and moved less.

Between RESP-BF and EDA-BF, the competence, tension, positive affect, sniper, enemy killed and game score showed very significant differences. Also, immersion, flow, negative affect, and movement exhibited significant differences. Overall, people scored poorly and felt low competence, immersion, and flow in the RESP condition. 


\begin{tabular}{|c|c|c|c|c|c|c|c|c|c|c|c|c|c|c|c|c|}
\hline & 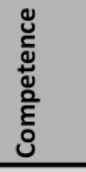 & 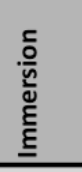 & 은 & $\begin{array}{l}\text { 은 } \\
\text { 든 } \\
\end{array}$ & 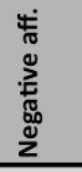 & 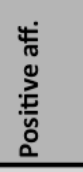 & 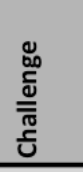 & 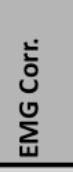 & $\begin{array}{l}\text { 은 } \\
0 \\
\text { 는 }\end{array}$ & 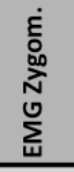 & 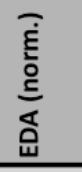 & 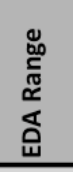 & 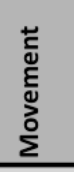 & $\frac{\grave{\Xi}}{\check{\Sigma}}$ & 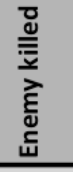 & 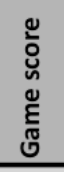 \\
\hline $\begin{array}{l}\text { SIM / } \\
\text { BF }\end{array}$ & $\begin{array}{l}2.9-3.3 / \\
2.3-3.6 \\
\end{array}$ & $\begin{array}{l}2.2-2.41 \\
2.1-2.6 \\
\end{array}$ & $\begin{array}{l}2.7-3.0 / \\
2.7-3.1 \\
\end{array}$ & $\begin{array}{l}2.3-2.8 / \\
2.2-3.1 \\
\end{array}$ & $\begin{array}{l}2.0-2.3 / \\
1.9-2.6 \\
\end{array}$ & $\begin{array}{l}2.4-2.8 / \\
2.3-2.9 \\
\end{array}$ & $\begin{array}{l}3.0-3.4 / \\
3.0-3.3 \\
\end{array}$ & $\begin{array}{l}24-271 \\
26-27 \\
\end{array}$ & $\begin{array}{c}18-24 / \\
18-20 \\
\end{array}$ & $\begin{array}{c}25-29 / \\
23-30\end{array}$ & $\begin{array}{l}3.1-3.21 \\
3.1-3.2 \\
\end{array}$ & $\begin{array}{l}41-44 / \\
40-42 \\
\end{array}$ & $\begin{array}{c}16-21 / \\
15-20 \\
\end{array}$ & $\begin{array}{l}91-110 / \\
86-117 \\
\end{array}$ & $\begin{array}{l}21-32 / \\
19-43 \\
\end{array}$ & $\begin{array}{l}16-24 / \\
14-34 \\
\end{array}$ \\
\hline $\begin{array}{c}\text { RESP SIM / } \\
\text { BF }\end{array}$ & $\begin{array}{l}2.9-3.1 / \\
2.3-2.9\end{array}$ & $\begin{array}{l}2.3-2.4 / \\
2.1-2.4 \\
\end{array}$ & $\begin{array}{l}2.7-2.8 / \\
2.7-2.9 \\
\end{array}$ & $\begin{array}{l}2.3-2.8 / \\
2.4-3.1 \\
\end{array}$ & $\begin{array}{l}2.1-2.3 / \\
2.0-2.6 \\
\end{array}$ & $\begin{array}{l}2.4-2.71 \\
2.3-2.5 \\
\end{array}$ & $\begin{array}{l}3.0-3.4 / \\
3.0-3.3\end{array}$ & $\begin{array}{l}24-271 \\
26-27 \\
\end{array}$ & $\begin{array}{c}18-24 / \\
18-20 \\
\end{array}$ & $\begin{array}{c}25-29 / \\
23-30 \\
\end{array}$ & $\begin{array}{l}3.1-3.21 \\
3.1-3.2 \\
\end{array}$ & $\begin{array}{l}41-44 / \\
40-41 \\
\end{array}$ & $\begin{array}{c}18-20 / \\
18-20 \\
\end{array}$ & $\begin{array}{c}\bullet \\
94-110 / \\
86-98 \\
\end{array}$ & $\begin{array}{c}21-27 / \\
19-21 \\
\end{array}$ & $\begin{array}{c}16-24 / \\
14-19 \\
\end{array}$ \\
\hline $\begin{array}{c}\text { EDA SIM / } \\
\text { BF }\end{array}$ & $\begin{array}{l}3.0-3.3 / \\
3.0-3.6 \\
\end{array}$ & $\begin{array}{l}2.2-2.4 I \\
2.2-2.6 \\
\end{array}$ & $\begin{array}{l}2.9-3.0 / \\
2.8-3.1 \\
\end{array}$ & $\begin{array}{l}2.3-2.5 / \\
2.2-2.4 \\
\end{array}$ & $\begin{array}{l}2.0-2.2 / \\
1.9-2.2 \\
\end{array}$ & $\begin{array}{l}2.8-2.8 / \\
2.8-2.9 \\
\end{array}$ & $\begin{array}{l}3.1-3.3 / \\
3.1-3.3 \\
\end{array}$ & $\begin{array}{l}26-271 \\
26-27 \\
\end{array}$ & $\begin{array}{c}20-221 \\
19-19 \\
\end{array}$ & $\begin{array}{c}26-28 / \\
25-27 \\
\end{array}$ & $\begin{array}{l}3.2-3.21 \\
3.1-3.2 \\
\end{array}$ & $\begin{array}{l}41-44 / \\
41-42 \\
\end{array}$ & $\begin{array}{c}16-21 / \\
15-19 \\
\end{array}$ & $\begin{array}{l}91-107 / \\
99-117 \\
\end{array}$ & $\begin{array}{l}\bullet \bullet \bullet \\
25-32 / \\
30-43 \\
\end{array}$ & $\begin{array}{l}\bullet \bullet \\
20-24 / \\
22-34 \\
\end{array}$ \\
\hline $\begin{array}{c}\text { RESP BF / } \\
\text { EDA BF }\end{array}$ & $\begin{array}{l}\bullet \bullet \bullet \\
2.3-2.9 / \\
3.0-3.6 \\
\end{array}$ & $\begin{array}{c}\bullet \\
2.1-2.4 / \\
2.2-2.6 \\
\end{array}$ & $\begin{array}{c}\bullet \\
2.7-2.9 / \\
2.8-3.1 \\
\end{array}$ & $\begin{array}{l}\bullet \bullet \bullet \\
2.4-3.1 / \\
2.2-2.4 \\
\end{array}$ & $\begin{array}{c}\bullet \\
2.0-2.6 / \\
1.9-2.2 \\
\end{array}$ & $\begin{array}{c}\bullet \bullet \bullet \\
2.3-2.5 / \\
2.8-2.9 \\
\end{array}$ & $\begin{array}{l}3.0-3.3 / \\
3.1-3.3 \\
\end{array}$ & $\begin{array}{l}26-271 \\
26-27 \\
\end{array}$ & $\begin{array}{c}18-20 / \\
19-19 \\
\end{array}$ & $\begin{array}{l}23-30 / \\
25-27\end{array}$ & $\begin{array}{l}3.1-3.21 \\
3.1-3.2 \\
\end{array}$ & $\begin{array}{l}40-41 / \\
41-42\end{array}$ & $\begin{array}{c}\bullet \bullet \\
18-20 / \\
15-19 \\
\end{array}$ & $\begin{array}{l}\bullet \bullet \bullet \\
86-98 / \\
99-117 \\
\end{array}$ & $\begin{array}{l}\bullet \bullet \bullet \\
19-21 / \\
30-43 \\
\end{array}$ & $\begin{array}{l}\bullet \bullet \bullet \\
14-19 / \\
22-34 \\
\end{array}$ \\
\hline $\begin{array}{c}\text { RESP SIM / } \\
\text { EDA SIM }\end{array}$ & $\begin{array}{c}\bullet \\
2.9-3.1 / \\
3.0-3.3 \\
\end{array}$ & $\begin{array}{l}2.3-2.4 I \\
2.2-2.4 \\
\end{array}$ & $\begin{array}{l}2.7-2.8 / \\
2.9-3.0 \\
\end{array}$ & $\begin{array}{l}2.3-2.8 / \\
2.3-2.5 \\
\end{array}$ & $\begin{array}{l}2.1-2.3 / \\
2.0-2.2 \\
\end{array}$ & $\begin{array}{c}\bullet \\
2.4-2.71 \\
2.8-2.8 \\
\end{array}$ & $\begin{array}{l}3.0-3.4 / \\
3.1-3.3 \\
\end{array}$ & $\begin{array}{l}24-271 \\
26-27 \\
\end{array}$ & $\begin{array}{l}18-24 / \\
20-22 \\
\end{array}$ & $\begin{array}{c}25-29 / \\
25-27 \\
\end{array}$ & $\begin{array}{l}3.1-3.21 \\
3.2-3.2 \\
\end{array}$ & $\begin{array}{l}41-44 / \\
41-44 \\
\end{array}$ & $\begin{array}{c}18-20 / \\
16-21 \\
\end{array}$ & $\begin{array}{l}94-110 / \\
91-107 \\
\end{array}$ & $\begin{array}{c}\bullet \\
21-27 \mid \\
25-32 \\
\end{array}$ & $\begin{array}{l}16-24 / \\
20-24 \\
\end{array}$ \\
\hline $\begin{array}{l}1^{\text {st }} \text { Round/ } \\
2^{\text {nd }} \text { Round }\end{array}$ & $\begin{array}{l}\bullet \bullet \bullet \\
2.3-3.01 \\
2.9-3.6\end{array}$ & $\begin{array}{l}2.4-2.6 / \\
2.1-2.3\end{array}$ & $\begin{array}{l}2.8-3.1 / \\
2.7-2.9\end{array}$ & $\begin{array}{c}\bullet \\
2.5-3.1 / \\
2.2-2.4\end{array}$ & $\begin{array}{c}\bullet \\
1.9-2.1 / \\
2.2-2.6\end{array}$ & $\begin{array}{l}2.3-2.8 / \\
2.5-2.9\end{array}$ & $\begin{array}{c}\bullet \\
3.3-3.4 / \\
3.0-3.1\end{array}$ & $\begin{array}{l}24-271 \\
26-27\end{array}$ & $\begin{array}{l}18-20 / \\
18-24\end{array}$ & $\begin{array}{c}25-30 / \\
23-29\end{array}$ & $\begin{array}{l}3.2-3.2 / \\
3.1-3.2\end{array}$ & $\begin{array}{c}41-44 / \\
40-41\end{array}$ & $\begin{array}{c}\bullet \\
19-21 / \\
15-18\end{array}$ & $\begin{array}{l}\bullet \bullet \\
86-99 / \\
98-117\end{array}$ & $\begin{array}{l}\bullet \bullet \\
19-30 / \\
21-43\end{array}$ & $\begin{array}{c}\bullet \bullet \\
14-22 / \\
19-34\end{array}$ \\
\hline
\end{tabular}

Table 1: Implicit condition comparison (phase 1 of the study) based on SPSS Linear Mixed-Model procedure

By contrast, there was little difference between the simulated and biofeedback stages overall. RESP-SIM and EDA-SIM showed differences in competence, positive affect, and enemies killed..

\section{Comparison between Implicit and Explicit Biofeedback}

Comparing phase 1 and phase 2 is not straightforward. The aforementioned carryover effects are significantly present in the comparison between phase 1 and phase 2 , as well as in comparison of the stages within phase 2, because RESP-BF and EDA-BF were always played in the respective order in phase 2 . For example, it is evident that there is some sort of "wow" factor for players once they hear about the biofeedback interaction within the game, because it is a novel gaming element and something of apparent interest to the experiment subjects.

The data in phase 1 showed only a few statistical differences between the simulation and biofeedback conditions. Psychophysiological data were especially insensitive to condition changes in all tests. Between phase 1 and phase 2, there was significant difference in EDA, EDA range ${ }^{5}$, and EMG corrugator supercilii (short. Cor.). EMG Cor. is usually interpreted as an indicator of negative flow or concentration. In the case of phase 2, EMG Cor. decreased significantly in the first stage (RESP-BF) and then increased significantly in the second (EDA-BF), but still it remained lower overall than in stages in phase 1.

Hence, according to psychophysiological data, the negative affect or concentration decreased for phase 2. The iGEQ value shows similar results. Negative affect decreased in phase 2 as it had earlier increased for round 2. However,

${ }^{5}$ EDA range is calculated from each stage's maximum and minimum EDA value. challenge increased for phase 2 and tension decreased. The tension decrease is in line with the EMG Cor. result. By far the most significant change in the GEQ results between phase 1 and phase 2 was in immersion. This score had decreased slightly from round 1 to round 2 but almost doubled for phase 2 .

In phase 2, only competence showed a difference between RESP-BF and EDA-BF. In phase 1, there were several significant differences between EDA-BF and RESP-BF, which disappeared for phase 2 . This is especially interesting if we also look at game score. As we noted in the previous section of the paper, we found significant difference in the game score between EDA-BF and RESP-BF stages. In phase 2, this difference disappeared. In fact, in phase 2, the game score was almost identical for RESP-BF (29 100) and for EDA-BF (29 200), which is significantly lower than with EDA-BF in phase 1's second round (35 000).

Finally, the EDA range was significantly greater in phase 2 EDA-BF than RESP-BF (0.56 and 0.34, respectively) and in phase 2 EDA-BF as compared to phase 1 EDA-BF. Both can be interpreted as indicating that during EDA-BF players changed their EDA levels consciously by manipulating their relaxation or arousal. Overall, according to the data there are many significant changes between the first-phase and second-phase EDA-BF and RESP-BF conditions.

The changes in the RESP condition are even greater than those with EDA. This is well shown by the transfer functions in figures 9 and 10. Minor changes are observable from Figure 9, but in Figure 10 (RESP) the changes are strong and clear. 


\begin{tabular}{|c|c|c|c|c|c|c|c|c|c|c|c|c|c|c|c|c|}
\hline & 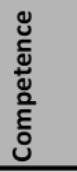 & 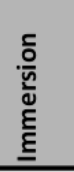 & 은 & 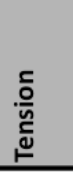 & 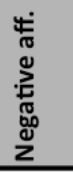 & 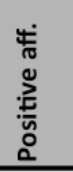 & 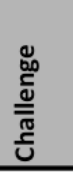 & 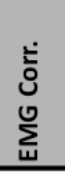 & 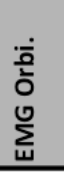 & 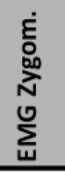 & 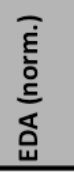 & 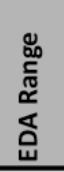 & 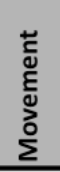 & ๕ั๊ & 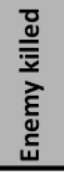 & 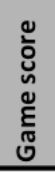 \\
\hline $\begin{array}{l}1^{\text {st }} \text { Phase / } \\
2^{\text {nd }} \text { Phase }\end{array}$ & $\begin{array}{l}2.3-3.6 / \\
3.0-3.4\end{array}$ & $\begin{array}{l}\bullet \bullet \bullet \\
2.1-2.6 / \\
3.7-3.7\end{array}$ & $\begin{array}{c}\bullet \bullet \\
2.7-3.1 / \\
3.2-3.2\end{array}$ & $\begin{array}{l}\bullet \bullet \bullet \\
2.2-3.1 / \\
1.7-1.7\end{array}$ & $\begin{array}{l}\bullet \bullet \bullet \\
1.9-2.6 / \\
1.5-1.5\end{array}$ & $\begin{array}{l}\bullet \bullet \\
2.5-2.9 / \\
3.0-3.2\end{array}$ & $\begin{array}{l}\bullet \bullet \bullet \\
3.0-3.4 / \\
3.6-3.7\end{array}$ & $\begin{array}{c}\bullet \bullet \\
24-27 / \\
20-22\end{array}$ & $\begin{array}{l}18-24 / \\
17-17 \\
\end{array}$ & $\begin{array}{c}23-30 / \\
20-20\end{array}$ & $\begin{array}{l}\bullet \bullet \bullet \\
3.1-3.21 \\
2.7-2.9\end{array}$ & $\begin{array}{l}40-44 / \\
34-64 \\
\end{array}$ & $\begin{array}{l}\bullet \bullet \bullet \\
15-21 / \\
12-13\end{array}$ & $\begin{array}{c}\bullet \\
86-117 / \\
91-97\end{array}$ & $\begin{array}{r}21-43 / \\
33-37 \\
\end{array}$ & $\begin{array}{r}14-34 / \\
27-27\end{array}$ \\
\hline $\begin{array}{l}\text { RESP } 1^{\text {st }} \mathrm{Ph} . / \\
2^{\text {nd }} \text { Phase }\end{array}$ & $\begin{array}{c}2.3-2.9 / \\
3.0\end{array}$ & $\begin{array}{c}\bullet \bullet \bullet \bullet \\
2.1-2.4 / \\
3.7\end{array}$ & $\begin{array}{c}\bullet \bullet \bullet \bullet \\
2.7-2.9 /\end{array}$ & $\begin{array}{c}\bullet \bullet \bullet \bullet \\
2.4-3.1 /\end{array}$ & $\begin{array}{c}\bullet \bullet \bullet \bullet \\
2.0-2.6 / \\
1.5\end{array}$ & $\begin{array}{c}\bullet \bullet \\
2.3-2.5 /\end{array}$ & $\begin{array}{c}\bullet \bullet \bullet \bullet \\
3.0-3.3 / \\
3.7\end{array}$ & $\begin{array}{c}\bullet \bullet \bullet \\
26-27 / \\
20\end{array}$ & $\begin{array}{c}18-20 / \\
17\end{array}$ & $\begin{array}{c}23-30 / \\
20\end{array}$ & $\begin{array}{c}\bullet \bullet \bullet \\
3.1-3.2 / \\
2.9\end{array}$ & $\begin{array}{c}40-41 / \\
34\end{array}$ & $\begin{array}{l}\bullet \bullet \bullet \\
18-20 / \\
13\end{array}$ & $\begin{array}{c}86-98 / \\
91\end{array}$ & $\begin{array}{l}\bullet \bullet \bullet \bullet \\
37\end{array}$ & $\begin{array}{c}\bullet \bullet \bullet \\
14-19 / \\
27\end{array}$ \\
\hline $\begin{array}{r}\text { EDA } 1^{\text {st }} \text { Phase / } \\
2^{\text {nd }} \text { Phase }\end{array}$ & $\begin{array}{c}3.0-3.6 / \\
3.4\end{array}$ & $\begin{array}{l}\bullet \bullet \bullet \bullet \\
2.2-2.6 / \\
3.7\end{array}$ & $\begin{array}{c}2.8-3.1 / \\
3.2\end{array}$ & $\begin{array}{c}\bullet \\
2.2-2.4 ! \\
1.7\end{array}$ & $\begin{array}{l}\bullet \bullet \bullet \\
1.9-2.2 / \\
1.5\end{array}$ & $\begin{array}{c}2.8-2.9 / \\
3.2\end{array}$ & $\begin{array}{l}\bullet \bullet \\
\begin{array}{l}\bullet .1-3.3 / \\
3.6\end{array}\end{array}$ & $\begin{array}{c}26-26 / \\
22\end{array}$ & $\begin{array}{c}19-19 / \\
17\end{array}$ & $\begin{array}{c}25-27 / \\
20\end{array}$ & $\begin{array}{c}\bullet \bullet \bullet \\
3.1-3.2 / \\
2.7\end{array}$ & $\begin{array}{c}\bullet \bullet \\
41-42 \\
64\end{array}$ & $\underset{12}{\bullet-19 /}$ & $\underset{99-110 /}{\bullet}$ & $\underset{33}{\bullet \bullet-43 /}$ & $\begin{array}{c}\bullet \\
22-34 \\
27\end{array}$ \\
\hline $\begin{array}{c}2^{\text {nd }} \mathrm{Ph} . \mathrm{RESP} / \\
2^{\text {nd }} \mathrm{Ph} . \mathrm{EDA}\end{array}$ & $3.0 / 3.4$ & $3.7 / 3.7$ & $3.2 / 3.2$ & $1.7 / 1.7$ & $1.5 / 1.5$ & $3.0 / 3.2$ & $3.7 / 3.6$ & $\underset{20 / 22}{\bullet}$ & $17 / 17$ & $20 / 20$ & $\stackrel{\bullet \bullet}{\bullet .9 / 2.7}$ & 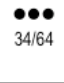 & $\stackrel{\bullet}{13 / 12}$ & $91 / 97$ & $33 / 37$ & $27 / 27$ \\
\hline
\end{tabular}

Table 2: Comparison between implicit (phase 1) and explicit (phase 2) conditions, and within explicit condition (phase 2)

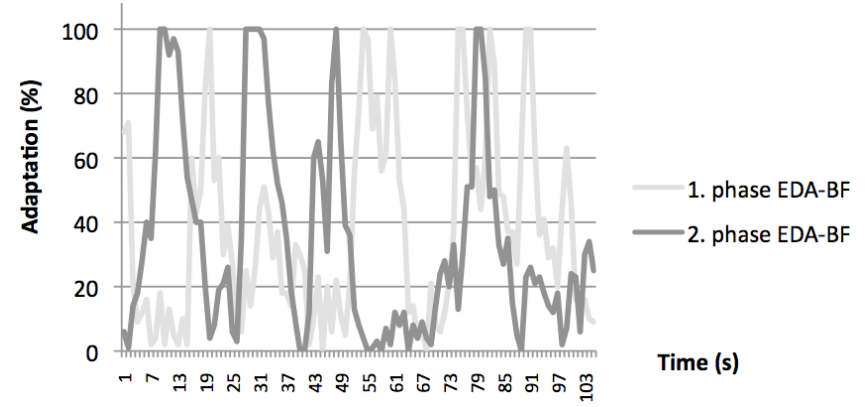

Figure 9: EDA-BF transfer function for phase 1. vs. phase 2

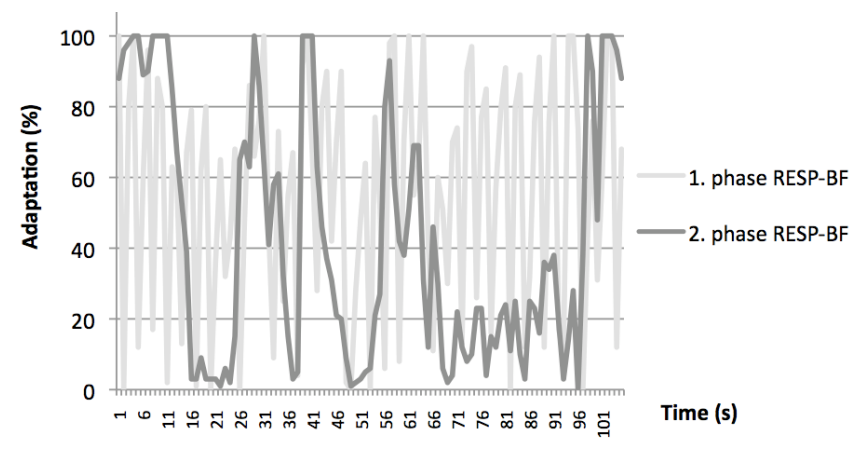

Figure 10: RESP-BF transfer function for phase 1 vs. phase 2

\section{DISCUSSION}

The data show that implicit biofeedback produces no effects in the RESP-BF condition. This result is shown to be valid by questionnaires, game measures, and psychophysiology measurements. In the EDA-BF condition, there are some significant differences. The transfer function design made the EDA-BF stage appear to produce the best score, but this fact did not lead to any changes in the emotional responses to the game play. Hence, the changes between simulation and biofeedback are because we did not succeed in creating accurate simulation for the EDA condition. However, that the EDA-BF and RESP-BF biofeedback conditions show differences, and the existence of a learning curve effect, demonstrates that the measures were sensitive. The differences between EDA-BF and RESP-BF are probably also results of variations in game controls, since comparison of RESP-BF and EDA-SIM shows slightly similar changes.

The explicit biofeedback condition showed many interesting changes. However, the findings in phase 2 are not counterbalanced. The difference between EDA-BF and RESP-BF changes from significant to nonexistent in GEQ measures and game measures. The great increase in the level of immersion, among other measures, indicates that people enjoyed playing in explicit biofeedback conditions. Figure 10, for example, shows that people were effectively using respiration signal as a controlled interaction channel in game interaction. Also, changes in EDA range and EDA normalized in the phase 2 EDA-BF stage, showing that they were able to use EDA at some level in interaction. However, each type of biosignal has distinct characteristics and usability issues, as demonstrated by the differences between RESP and EDA results in our experiments. Controlling EDA is harder and makes players perform worse in the explicit condition than the implicit, whereas game success improved dramatically in the explicit RESP stage.

This co-adaptation can increase the fun of playing but can also make the design of balanced game mechanics with biofeedback highly challenging. If the biofeedback adaptation does not affect the competitive side of game mechanics but is designed to increase the immersion or believability of the game in some non-competitive manner, the issues of manipulation may not be as significant.

Good games are composed of delicate synthesis of the components, creating a pleasant game balance and challenge for players. Therefore, defining suitable game design patterns [2] for biofeedback adaptation is the key issue in this kind of game development. The objective in our experiment was to design biofeedback interaction that would strongly affect the game playing experience while simultaneously being meaningful from the game-play perspective. We have explored character identification as a game design pattern, and the growth in measurable player immersion in the case of explicit biofeedback demonstrates that this adaptation is 
effective in the context of first-shooter games in the explicit biofeedback condition. It can also be hypothesized that similar increased effects of immersion could be achieved in other contexts, such as 3D virtual environments.

\section{CONCLUSIONS}

In our tests of adaptive biofeedback game, we have been able to produce meaningful gaming patterns and game adaptations. However, we observed that introducing biofeedback adaptation significantly increases the complexity of the game design tasks involved. From quantitative data, we can recommend the use of explicit biofeedback in first-person shooter games to increase the quality of the gaming experience. Implicit biofeedback appears non-responsive in terms of experience, and complicated to design. Explicit biofeedback is responsive, players are able to manipulate the biosignals, and explicit biofeedback game interaction appears to increase immersion and challenge. Also, it is much easier to design explicit biofeedback manipulations than implicit biofeedback. Even though the explicit biofeedback part of the study had some structural problems, the empirical data still indicate that players are able to manipulate biosignals such as EDA consciously, with a relatively brief learning period.

\section{REFERENCES}

1. Bersak, D., McDarby, G., Augenblick, N., McDarby, P., McDonnell, D., McDonald, B., and Karkun, R. (2001). Intelligent Biofeedback Using an Immersive Competitive Environment. Designing Ubiquitous Computing Games Workshop at UbiComp 2001, Atlanta, Georgia, USA.

2. Björk, S. and Holopainen, J. (2004). Patterns in Game Design. Hingham, Mass., USA: Charles River Media. 423 p. ISBN: 9781584503545.

3. Dekker, A. and Champion, E. (2007). Please Biofeed the Zombies: Enhancing the Gameplay and Display of a Horror Game Using Biofeedback. In: Proceedings of DiGRA 2007: Situated Play, September 24-28, 2007, Tokyo, Japan.

4. Fairclough, S. H. (2007). Psychophysiological Inference and Physiological Computer Games. Brainplay '07: Brain-Computer Interfaces and Games. Advances in Computer Entertainment, Salzburg, Austria.

5. Freeman, D. (2004). Creating Emotion in Games: The Craft and Art of Emotioneering. USA: New Riders. 539 p. ISBN: 1-5927-3007-8.

6. Gilleade, K. M., Dix, A., and Allanson, J. (2005). Affective Videogames and Modes of Affective Gaming: Assist Me, Challenge Me, Emote Me. Proceedings of DiGRA 2005: Changing Views Worlds in Play, June 16-20, 2005, Vancouver, Canada.
7. Grodal, T. (2000). Video Games and the Pleasures of Control. In: D. Zillmann and P. Vorderer (eds.), Media Entertainment: The Psychology of Its Appeal (pp. 197-212). Mahwah, New Jersey, USA: Lawrence Erlbaum Associates.

8. Hefner, D., Klimmt, C., and Vorderer, P. (2007). Identification with the Player Character As Determinant of Video Game Enjoyment. In: L. Ma, R. Nakatsu, \& M. Rauterberg (eds.), International Conference on Entertainment Computing 2007 (Lecture Notes in Computer Science 4740, pp. 39-48). Berlin: Springer.

9. Kim, J. and André, E. (2008). Four-Channel Biosignal Analysis and Feature Extraction for Automatic Emotion Recognition. In: Proceedings of BIOSTEC 2008, A. Fred, J. Filipe, \& H. Gamboa (eds.). CCIS 25, pp. 265-277, Berlin: Springer-Verlag.

10. Lang, P. J. (1995). The Emotion Probe. Studies of Motivation and Attention. American Psychologist, 50, 372-385.

11. Lindley, C. and Sennersten, C. (2008). Game Play Schemas: From Player Analysis to Adaptive Game Mechanics. International Journal of Computer Games Technology, 2008, Article ID 216784.

12. Picard, R. W., Vyzas, E., and Healey, J. (2001). Toward Machine Emotional Intelligence: Analysis of Affective Physiological State. IEEE Transactions on Pattern Analysis and Machine Intelligence, 23, 10, 1175-1191. ISSN: 0162-8828.

13. Ravaja, N., Saari, T., Laarni, J., Kallinen, K., Salminen, M., Holopainen, J., and Järvinen, A. (2005). The Psychophysiology of Video Gaming: Phasic Emotional Responses to Game Events. In: Proceedings of DiGRA 2005 Conference: Changing Views - Worlds in Play, Vancouver, Canada. June 16-20, 2005, pp. 1-13.

14. Scherer, K. R. (1993). Neuroscience Projections to Current Debates in Emotion Psychology. Cognition and Emotion, 7, 1-41.

15. Tijs, T. J., Brokken, D., and Ijsselsteijn, W. A. (2008). Dynamic Game Balancing by Recognizing Affect. Fun and Games 2008 Conference. Eindhoven, Netherlands. October 20-21, 2008, pp. 88-93.

16. Vorderer, P., Hartmann, T., and Klimmt, C. (2003). Explaining the Enjoyment of Playing Video Games: The Role of Competition. In: D. Marinelli (ed.), Proceedings of the 2nd International Conference on Entertainment Computing (ICEC 2003) (pp. 1-8). Pittsburgh, Penn., \& New York, New York, USA: ACM. 